\section{Deeper insights into cell death and survival}

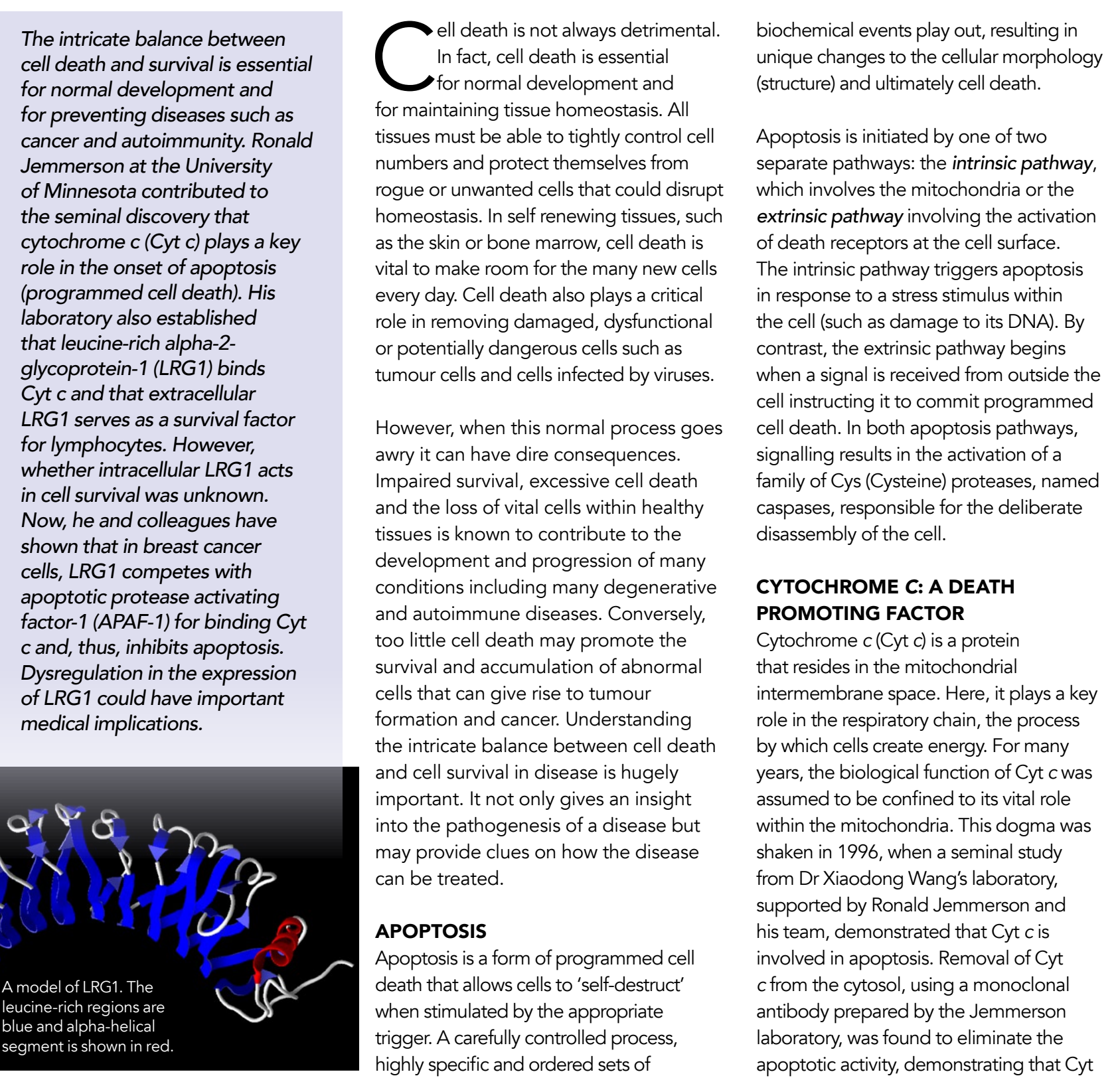
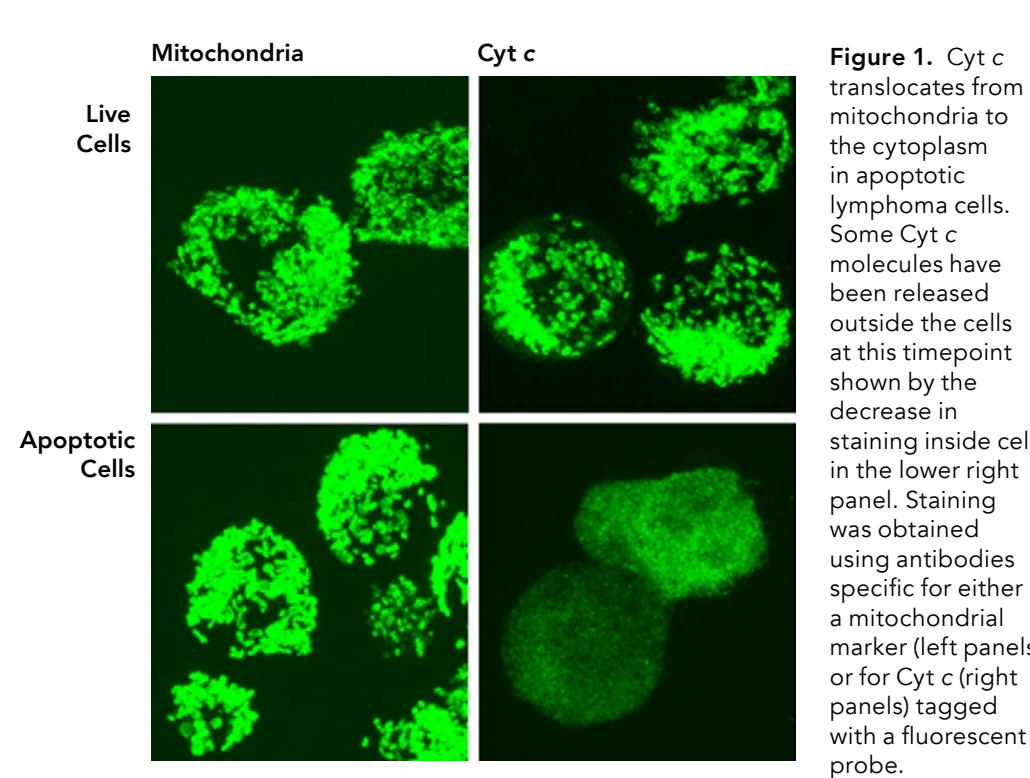

c, and not a contaminant, was responsible for the proapoptotic activity. A second laboratory, specific for a short section of the tail end of Cyt c provided the tool in an assay in which cellular proteins lose their normal three-dimensional structure, to demonstrate that Cyt
c moves to the cytoplasm during apoptosis.

These findings were quickly reproduced in other laboratories and to date the study has been cited in over 6,000 publications. It's now known that follow Cy release into the cytoplasm of the cell, Cyt c binds apoptotic protease activating factor-1 (APAF-1) where it promotes the formation of the apoptosome and
subsequent caspase activation to bring subsequent caspoptosis.

The discovery that Cyt c plays a role was profound and has had a marked impact across the fields of biology and surrounding the potentia of Cytcas a clinical marker to detect aberrant apoptosis, with a view to exploit tits role in cell death as potential therapy.

The discovery that Cyt c plays a role in both the life and death of a cell was profound. LRG1: A SURVIVAL FACTOR Elevated levels of Cyt c in the blood are reported in patients with inflammatory conditions or in patients recovering from chemotherapy treatment for cancer. Thus, Jemmerson and his team set out quantify levels of Cyt cin sera of patients these attempts were hindered by a component in serum that blocked the detection of Cyt c. The team identified

Keen to explore the role of LRG1 further, and especially its effect on binding to Cyt c, the Jemmerson laboratory carried out experiments using both human and mouse lymphocytes (a type of immune cell). Notably, they demonstrated that IRG acts as a survival protein, protecting the cells from apoptosis induced by hypothesised that LRG1 may protect cells from apoptosis by sequestering Cyt c. In fact, the team discovered that the mechanism of protection by LRG1 appears to involve active signalling. That 1, when LRG binds to Cyt cit seems deliver a that mouse LRG1 was more effective han human LRG1 in protecting mouse ymphocytes against extracellular Cyt induced apoptosis, suggesting that a

$$
\begin{aligned}
& \text { species-specific component } \\
& \text { pars }
\end{aligned}
$$

plays a role in transmitting the survival signal to the cell. To date this component, perhaps be identified.

In a recent publication in the journal Apoptosis, Jemmerson and colleagues reported that LRG1 is present in the cytoplasm of a breast cancer cell line, where it protects against apoptosis by binding cytoplasmic Cyt c and preventing otherise initite cell deeth in this study, MCF-7 breast cancer cells that had either increased or decreased expression of LRG1 were exposed to the apoptosis
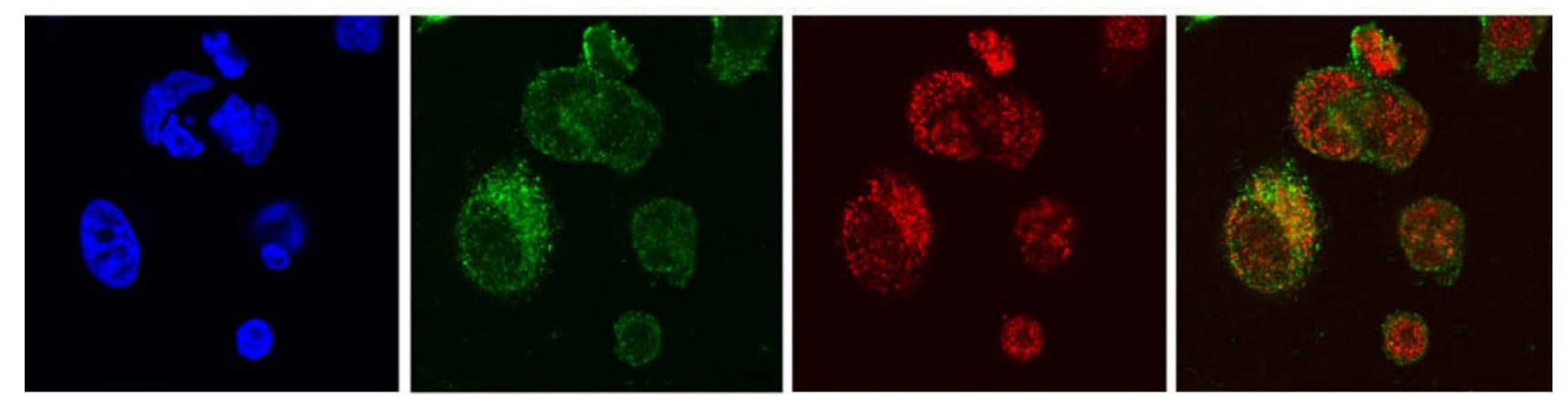

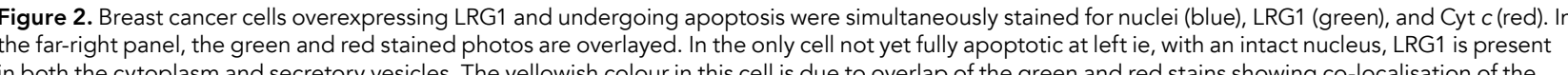

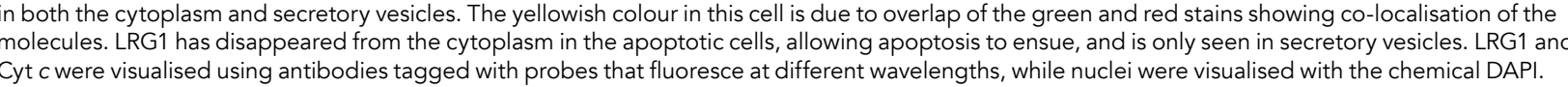


INTRACELLULAR SURVIVAL PROMOTION

Pre-apoptotic cytoplasm

High affinity binding of $L R G 1$ sequesters
Cyt c present in the cytoplasm blocking
binding to APAF-1 and apoptosis induction

inducing agent hydrogen peroxide and the effect on cell survival was examined. THE ACTION OF LRG

The expression was increased in cells. Were more resistant to hydrogen peroxide induced apoptosis than control cells. Conversely, cells with decreased LRG1 peroxide induced apoptosis. Interestingly, although more Cyt c was detected in the than in the cytosol of either control or cells with lower levels of LRG1, the Cyt c was bound to LRG1, not to APAF-1, and therefore unable to induce apoptosis. protection offered by extracellular LRG previously reported for lymphocytes, whereby prt of the IRG1 structance whereby part of the LRG1 structure physically blocks Cyt c from binding higher affinit than does APAF-1, it may serve as an effective trap to capture $C y$ cthat would appear in the cytoplasm. It's thought that LRG1 may normally protect against transient increases in Cyt $c$ released to the cytoplasm in the absence of committed apoptotic signalling.

Once committed apoptotic signalling occurs, in this case, following exposure of the cells to hydrogen peroxide, LRG1 was found to be degraded, allowing Cyt $c$ to bind APAF- 1 and activate the intrinsic apoptotic pathway. Thus, in the absence of committed apoptotic signalling, cell survival is affected by the chinding to APAF-1. As a consequeyt dysinging to APAF-1. As a consequence,

EXTRACELLULAR SURVIVAL PROMOTION

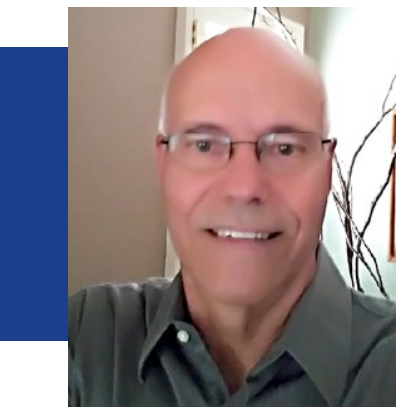

Behind the Research

Professor Ron Jemmerson

E: jemme001@umn.edu T: +1 760-671-7081

W: https://med.umn.edu/bio/microbiology/ronald-jemmerson

In a series of elegant genetic studies, levels were more sensitive to hydrogen cytosol of cells with higher LRG1 levels Thus, in contrast to the mechanism of the mechanism of protection in this case

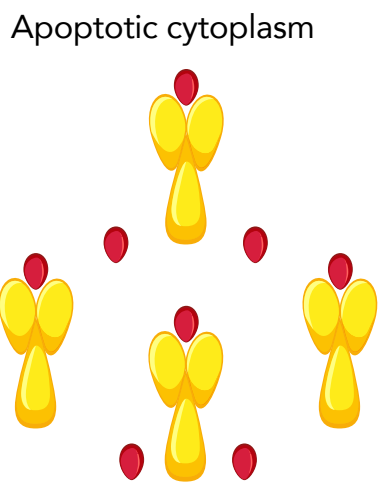

After LRG1 is degraded APAF-1

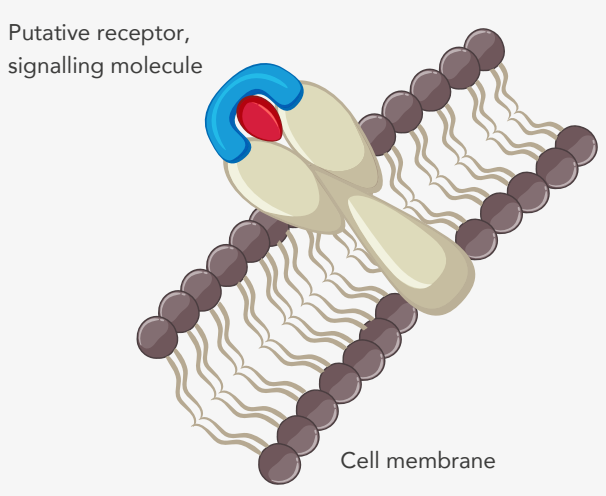

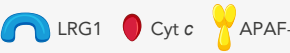

of LRG1 in cells may support the development of cancer and exacerbate inflammation and autoimmunity

In addition to its important role in apoptosis, it's known that Cyt c can pattern (DAMP), molecules released by damaged or dying cells capable of alarming the immune system to any danger Thus, Cytc appears to perform distinct functions depending upon its $c$ resides in the mitochondria playing a vital role in the respiratory chain. In response to pro-apoptotic signalling, its

ranslocation to the cytoplasm initiates the process of cell death. Finally, upon loss of membrane integrity and its release into and failtating the repair of host issung

The work of Ronald Jemmerson and his of Cyt c to LRG1 promotes cell sunival. usual location in mitochondria, either within or outside the cell, and in concert with its binding partners, APAF-1 or LRG1, it plays a pivotal role in the balance between cell death and survival.

LRG1 acts as a survival protein, protecting the cells from apoptosis induced by Cyt $c$.

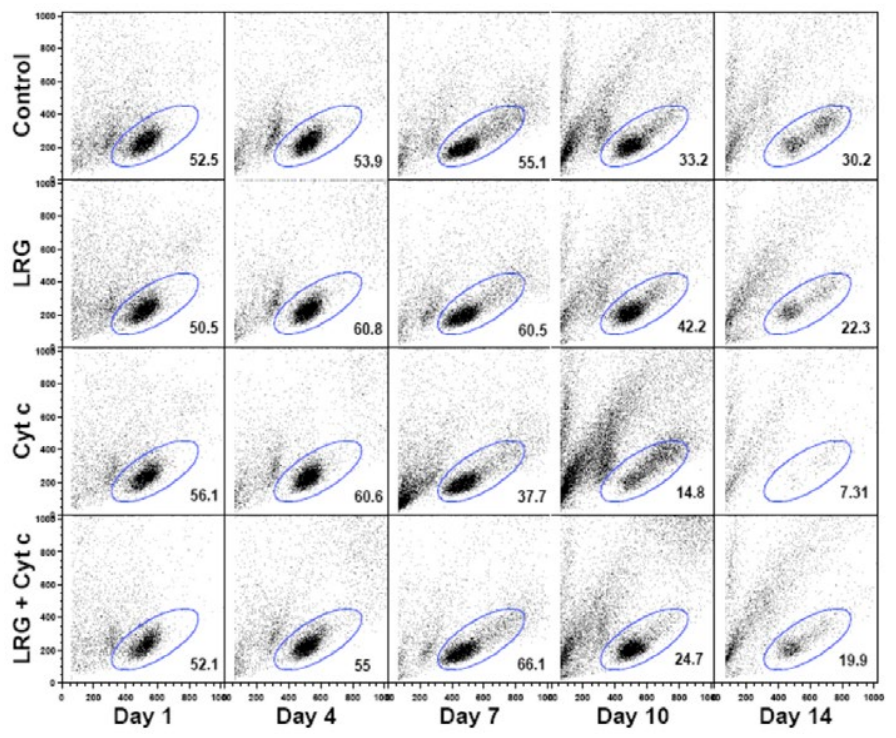

Figure 3. Human lymphocytes were individually assessed tor their light scattering properties in flow cytometry. Here, forward scatter (X-axis and side scatter (Y-axis) are plotted. The number in each
panel is the eprcentage of viable cells shown within the blue oval. The e cells were cultured in LRG1-free panel is the percentage of viable cells shown within the blue oval. The cells were cultured in LRG1-free
medium. At day 7 there was a marked decrease in the epr centage of cells treated with Cyt cthat were
viable compared to cells cultured in other conditions. LRG1 is shown to protect the iymphocytes from location. Under normal conditions, Cyt the extracellular environment it may have Thus, when Cyt c is displaced from its

\section{Research Objectives}

Ron Jemmerson contributed to the ground-breaking study showing that cytochrome $c($ Cyt c) translocates from mitochondria to the cytoplasm during apoptosis and plays a role in its onset. This inspired further study of Cyt $c$ as a promoter and indicator of cell death.

\section{Detail}

Address

Department of Microbiology and Immunology

Bio

Ron Jemmerson received his BA from Western Maryland Biology, Northwestern University, 1978 He was a postdoctoral fellow Research Institute of Scripps Clinic, La J CA, before holding the position of faculty member at the Department of Microbiology and Immunology, University of Minnesota since 1985, where he is currently Professor emeritus.

Funding

Medical Foundation

- University of Minnesota Graduate School

National

Neurological Disorders and Stroke

National Science Foundation (USA)

Collaborators

(University of Minnesota)

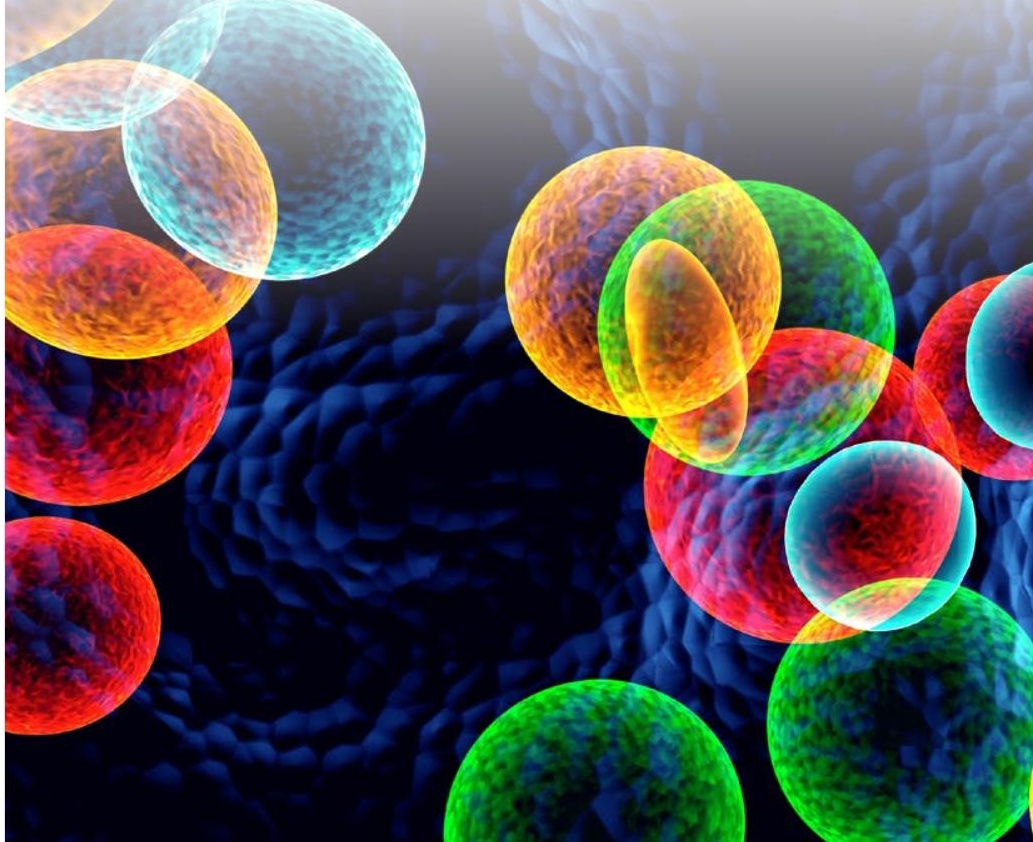

\section{References}

Liu, X Kim, CN Yang, J Jemmerson, R and Wang, X (1996). Induction of apoptotic program in cell-free extracts: Requirement for dATP and cytochrome c. Cell 86: 147-157

Jemmerson, R LaPlante, B and Treeful, A (2002). Release of intact, monomeric cytochrome $\mathrm{c}$ from apoptotic and necrotic cells. Cell Death and Differentiation 9:538-548

Cummings, C Walder, J Treeful, A and Jemmerson, R (2006). Serum leucine-rich alpha-2-glycoprotein-1 binds cytochrome $c$ and inhibits antibody detection of this Apoptosis 11: 1121-1129

Codina, R Vanasse, A Kelekar, A Vezys, V and Jemmerson $R$ (2010). Cytochrome $c$-induced lymphocyte death from the outside in: inhibition by serum leucine-rich alpha-2glycoprotein-1. Apoptosis 15: 139-152

Jemmerson, R Staskus, K Higgins, L Conklin, K and Kelekar, A (2021). Intracellular leucine-rich alpha-2-glycoprotein-1 competes with Apaf-1 for binding cytochrome $c$ in protecting MCF 7 breast cancer cells from apoptosis.

\section{Personal Response}

Is it possible to exploit/manipulate the binding of LRG-1 to Cyt c to modulate the life or death of a cell as a potentialtherapy for those diseases associated with

II By testing 1,200 commercially available off-patent compounds for blocking LRGI capture by Cyt cin an candidates that blocked LRG1 capture but did not bind Cyt $c$ and, thus, should not interfere with APAF-1 activation. The exceptionally high affinity of LRG1 for Cyt c reported by others will present a challenge finding a competing compound efectivesyste Acaly fibcong a comrations possible for impacting extracellular LRG functions as shown by John Greenwood's research group in inhibiting
pathogenic neovascularisation induced by LRG1.

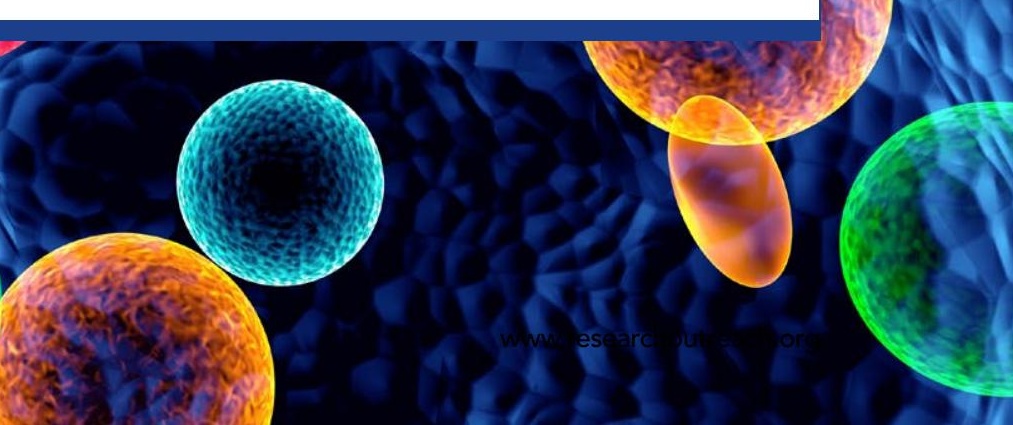

